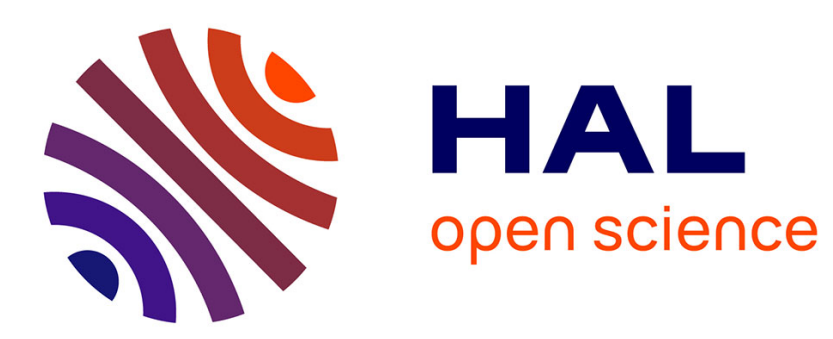

\title{
Sliding Modes Observers for Vehicle Dynamics and Variable Structure Automatic Systems (SMO-VSAS)
}

N K K Msirdi, Sirdi B Jaballah, H. Nasser, A. Naamane

\section{To cite this version:}

N K K Msirdi, Sirdi B Jaballah, H. Nasser, A. Naamane. Sliding Modes Observers for Vehicle Dynamics and Variable Structure Automatic Systems (SMO-VSAS). 2010 11th International Workshop on Variable Structure Systems (VSS), Jun 2010, Mexico city, Mexico. hal-02496394

\section{HAL Id: hal-02496394 \\ https://hal.science/hal-02496394}

Submitted on 3 Mar 2020

HAL is a multi-disciplinary open access archive for the deposit and dissemination of scientific research documents, whether they are published or not. The documents may come from teaching and research institutions in France or abroad, or from public or private research centers.
L'archive ouverte pluridisciplinaire HAL, est destinée au dépôt et à la diffusion de documents scientifiques de niveau recherche, publiés ou non, émanant des établissements d'enseignement et de recherche français ou étrangers, des laboratoires publics ou privés. 


\title{
Sliding Modes Observers for Vehicle Dynamics and Variable Structure Automatic Systems (SMO-VSAS)
}

\author{
N.K. M'Sirdi \\ B. Jaballah \\ H. Nasser \\ A. Naamane \\ LSIS, CNRS UMR 6168. Domaine Universitaire Saint Jrme, Avenue Escadrille Normandie - Niemen 13397. \\ Marseille Cedex 20. France.nacer.msirdi@lsis.org and Polytech Marseille, 13013 Marseille, France.
}

\begin{abstract}
This paper will show how to handle modeling of vehicles to get efficient and good Sliding Mode Observers. A car model with $16 \mathrm{DoF}$ is decomposed for partial state observation with SMO. This decomposition method may lead, in VSAS, to good estimates of different kind variables and inputs (subsystems states, environment and ground variables, interfaces variables, connections and constraint variables). Robust Sliding Mode Observers and unknown input estimations are developed.
\end{abstract}

\section{INTRODUCTION}

1 In literature, many studies deal with vehicle modeling [1][2][3]. Vehicle dynamics can be represented by approximate models which are either too much simplified to be realistic or complex and too much wide involving big simulation softwares. In nature they have a variable structure (VSAS) but in literature, their properties are never detailed nor their passivity property emphasized. This kind of systems, like in general VSAS, are composed with many passively coupled subsystems: wheels, motor and braking control system, suspensions, steering, more and more inboard and embedded electronics. There are several non linear parts in VSAS, which are coupled. These coupling may be time varying and non stationary. Approximations have to be made carefully regarding to the desired application, see eg [4].

In our previous works a good nominal vehicle model with 16 DOF have been validated in a simulator, we developed for a French car type (Peugeot 406), [2]. Several interesting applications was successful and have been evaluated by use of this simulator before actual results [5]. We have also considered this modeling for estimation of unknown inputs [6], interaction parameters and exchanges of VSAS with environment [7]. This approach has been used successfully also for heavy vehicles [8].

In this paper the car model is revisited as VSAS and structured for estimation of inputs and diagnosis. We split the model in five subsystems [9] and then show and justify the rationale behind the successful splitting to get simple and efficient partial (sub) models. The subsystems and the overall system obey the passivity property. This feature, like in Bond Graphs modeling emphasize the energy flow in VSAS and exchanges between the system parts and also with the environment. After the structure and model analysis, we consider estimation of the partial states for diagnosis and motion control in the vehicle.

\footnotetext{
${ }^{1}$ This work was supported by GTAA of GdR MACS (CNRS) and the French Carnot Institute "IC STAR". Acknowledgement is addressed to GII department of Polytech Marseille.
}

Robust estimations are necessary to be able to obtain good evaluation of the VSAS driving situation at each time instant.

\section{Vehicle Model Parametrization}

\section{A. The Nominal global model}

The Nominal model of a car vehicle with uncertainties has been developed assuming the car body rigid and pneumatic contact permanent and reduced to one point for each wheel (see eg [7] [9] [10]). It is composed by 4 non linear equations: one for the mechanical dynamics of the vehicle (equation (1)), one for interface torques and forces (vehicle inputs equation (2)), one for the contact with environment (equation (3)) and the last one for the environment normal reactions (equation(4)). $\tau$ is the input vector composed by torques and forces applied to the vehicle.

$$
\begin{aligned}
\tau & =M(q) \ddot{q}+C(q, \dot{q}) \dot{q}+V(q, \dot{q})+G(q)+\eta_{o}(t, q, \dot{q}) \\
\tau & =\Gamma_{e}+\xi J\left(x_{1}\right)^{T} F \\
\dot{F} & =f\left(\alpha, \lambda, q, F_{N}\right)+e(t) \\
F_{N} & =h\left(l_{f}, l_{r}, h, g, \dot{v}_{x}, \dot{v}_{y}, q, x_{\text {road }}, \beta, \gamma\right)
\end{aligned}
$$

The generalized coordinates vector $q \in R^{16}$ is defined as $q^{T}=\left[x, y, z, \theta_{z}, \theta_{y}, \theta_{x}, q_{31}, q_{32}, q_{33}, q_{34}, \delta_{3}, \delta_{4}, \varphi_{1}, \varphi_{2}, \varphi_{3}, \varphi_{4}\right]$ where $x, y$, and $z$ represent displacements. Angles of roll, pitch and yaw are $\theta_{x}, \theta_{y}$ et $\theta_{z}$ respectively. The suspensions elongations are noted $q_{3 i}$ : $(i=1 . .4) . \delta_{i}$ : stands for the steering angles. $\varphi_{i}$ : are angles of wheels rotations $(i=1 . .4$.). $\dot{q}, \ddot{q} \in R^{16}$ are respectively velocities and corresponding accelerations. The state vector is $x^{T}=\left(x_{1}^{T}, x_{2}^{T}\right)=\left(q^{T}, \dot{q}^{T}\right)$. The random part in forces $e(t)$ is due to neglected and uncertain dynamics in wheels contact. the variable $\xi$ is equal to unity when the corresponding wheel is in contact with the ground and zero if not.

Equation (1) describes the dynamics of the vehicle excited by the external forces of equation (2) which stands as interface with environment. $\xi J\left(x_{1}\right)^{T} F$ represents the environment reactions and control inputs are in $U=M\left(x_{1}\right)^{-1} \Gamma_{e}$. The $\eta_{o}(t, q, \dot{q})$ represent external perturbations, uncertainties and neglected dynamics.

The gravity term is $G(q) . V(q, \dot{q})=\xi\left(K_{v} \dot{q}+K_{p} q\right)$ are Suspensions forces (with respectively damping and stiffness matrices $\left.K_{v}, K_{p}\right)$; We can have also in $V(q, \dot{q})$ Coulomb friction which contain relay terms depending on velocities and positions (non linear frictions). 
Equation (4) gives the environment reaction forces to vehicle posture. The latter forces produce the wheel-ground contact forces (3).

\section{B. Mechanical Model Properties}

Then we have the state space representation of our VSAS (see [9])

$$
\begin{aligned}
& \dot{x}_{1}=x_{2} \\
& \dot{x}_{2}=f\left(x_{1}, x_{2}\right)+M^{-1} \xi J^{T} F+U-\eta\left(x_{1}, x_{2}\right) \\
& y=h(x) \\
& \tau=\Gamma_{e}+\xi J\left(x_{1}\right)^{T} F, \quad U=M^{-1} \Gamma_{e} \\
& \dot{F}=f\left(\alpha, \lambda, q, F_{N}\right)+e(t) \\
& F_{N}=h\left(l_{f}, l_{r}, h, g, \dot{v}_{x}, \dot{v}_{y}, q, x_{\text {road }}, \beta, \gamma\right)
\end{aligned}
$$

$f\left(x_{1}, x_{2}\right)=-M\left(x_{1}\right)^{-1}\left(C\left(x_{1}, x_{2}\right) x_{2}+V\left(x_{1}, x_{2}\right)+G(q)\right)$, is the nominal dynamics assuming normal driving situation. $\eta\left(x_{1}, x_{2}\right)=M\left(x_{1}\right)^{-1} \eta_{o}\left(t, x_{1}, x_{2}\right)$ are the model uncertainties, input perturbations and neglected dynamics in VSAS.

The mechanical model part (1) is passive and has several interesting properties, which are well known and extensively used in robotics. We note that the system (1-4) can be cast in the Equivalent Passive Feedback Scheme (EPFS) shown in figure (1)

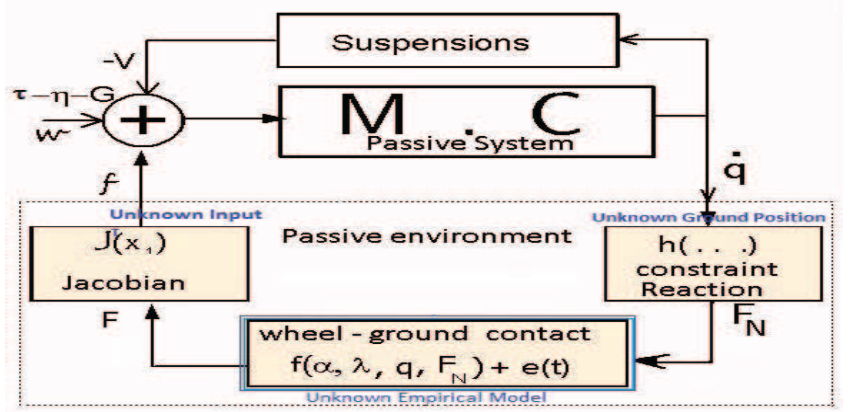

Figure 1. Vehicle dynamics in a Passive Feedback Equivalent Scheme.

Properties: Matrices $M$ and $C$ are such as (see [11][2][12]):

1) The inertia matrix $M(q)$, of dimensions $16 \times 16$, is Symmetric Positive Definite(SPD).

2) $N=\dot{M}(q)-2 C(q, \dot{q})$ is skew symmetric, i.e $\forall v \in R^{n}$, $v^{T} N v=0$ or $N=-N^{T}$.

3) $C(q, \dot{q}) \epsilon=C(q, v) v-\Pi(q, v) \epsilon$, with $\epsilon=v-\dot{q}$ and $\Pi(q, v)=\frac{\partial}{\partial x_{2}}\{C(q, \dot{q}) \dot{q}\}_{\dot{q}=v}$.

4) The input torque $w=\tau-\eta_{0}-G(q)$ is composed by two parts one from actuators (driver) and a second from environment reaction and perturbations.

5) All the perturbation terms $\eta_{i}$ (coupling, neglected $d y$ namics), are bounded: $\exists k_{i}>0, i=1, . .5$, such and as $\left|\eta_{i}\right|<k_{i} \forall t \in R$

\section{Coupled sub models}

The VSAS model (1) is then split in 5 equations corresponding respectively to chassis translations, chassis rotations, Suspensions elongations, wheel steering and wheel rotations, with as positions $q_{1}, q_{2}, q_{3}, q_{4}$ and $q_{5}$. The fastest motions are wheel rotations and the slowest ones are the chassis translations and rotations with wheels steering.

Owing to the structure of his matrices $M\left(x_{1}\right)$ and $C\left(x_{1}, x_{2}\right)$, we can split the model (1) in five parts as follows (see for details [13], [4]) with $q^{T}=\left[q_{1}^{T}, q_{2}^{T}, q_{3}^{T}, q_{4}^{T}, q_{5}^{T}\right]$.

$$
\begin{aligned}
& q_{1}^{T}=[x, y, z] \quad q_{2}^{T}=\left[\theta_{z}, \theta_{y}, \theta_{x}\right] \quad q_{4}^{T}=\left[\delta_{3}, \delta_{4}\right] \\
& q_{3}^{T}=\left[q_{31}, q_{32}, q_{33}, q_{34}\right] \quad q_{5}^{T}=\left[\varphi_{1}, \varphi_{2}, \varphi_{3}, \varphi_{4}\right]
\end{aligned}
$$

The 16 Degrees of Freedom model (eq(1)) is rewritten:

$$
\begin{gathered}
{\left[\begin{array}{c}
F_{T} \\
F_{R} \\
F_{S} \\
U_{4} \\
U_{5}
\end{array}\right]=\left[\begin{array}{lllll}
\bar{M}_{1,1} & \bar{M}_{1,2} & \bar{M}_{1,3} & 0 & 0 \\
\bar{M}_{2,1} & \bar{M}_{2,2} & \bar{M}_{2,3} & \bar{M}_{2,4} & \bar{M}_{2,5} \\
\bar{M}_{3,1} & \bar{M}_{3,2} & \bar{M}_{3,3} & 0 & 0 \\
0 & \bar{M}_{4,2} & 0 & \bar{M}_{4,4} & 0 \\
0 & \bar{M}_{5,2} & 0 & 0 & \bar{M}_{5,5}
\end{array}\right]\left[\begin{array}{c}
\ddot{q}_{1} \\
\ddot{q}_{2} \\
\ddot{q}_{3} \\
\ddot{q}_{4} \\
\ddot{q}_{5}
\end{array}\right]+} \\
+\left[\begin{array}{lllll}
0 & \bar{C}_{12} & \bar{C}_{13} & 0 & 0 \\
0 & \bar{C}_{22} & \bar{C}_{23} & \bar{C}_{24} & \bar{C}_{25} \\
0 & \bar{C}_{32} & \bar{C}_{33} & 0 & 0 \\
0 & \bar{C}_{42} & 0 & 0 & \bar{C}_{45} \\
0 & \bar{C}_{52} & 0 & \bar{C}_{54} & 0
\end{array}\right]\left[\begin{array}{l}
\dot{q}_{1} \\
\dot{q}_{2} \\
\dot{q}_{3} \\
\dot{q}_{4} \\
\dot{q}_{5}
\end{array}\right]+\left[\begin{array}{c}
\eta_{1} \\
\eta_{2} \\
\eta_{3} \\
\eta_{4} \\
\eta_{5}
\end{array}\right]+\left[\begin{array}{c}
V_{1} \\
V_{2} \\
V_{3} \\
V_{4} \\
V_{5}
\end{array}\right]
\end{gathered}
$$

$F_{T}, F_{R}$ and $F_{S}$ are forces or torques corresponding to translation, rotations of the chassis and excitation of suspensions respectively. They come from $J\left(x_{1}\right)^{T} F . U_{4}$ is the steering control and $U_{5}$ stands for brake and motor torques. The terms $V_{i}$ contain the remaining part of equation (1) accounting for frictions, suspensions stiffness, damping and gravity.

1) Dynamics of the chassis $\Sigma_{1}$ : From the global system we keep the two first equations (7) for translations and rotations.

$$
\begin{aligned}
& F_{1}=M_{1}\left[\begin{array}{c}
\ddot{q}_{1} \\
\ddot{q}_{2}
\end{array}\right]+C_{1}\left[\begin{array}{c}
\dot{q}_{1} \\
\dot{q}_{2}
\end{array}\right]+\left[\begin{array}{c}
V_{1} \\
V_{2}
\end{array}\right]+\nu_{1} \\
& F_{1}=\left[\begin{array}{c}
F_{T} \\
F_{R}
\end{array}\right] ; \quad \nu_{1}=\left[\begin{array}{c}
\eta_{c}^{1} \\
\eta_{c}^{2}
\end{array}\right] ; \quad J_{1}^{T}=\left[\begin{array}{cc}
J_{1,1} & J_{1,2} \\
J_{2,1} & J_{2,2}
\end{array}\right] \\
& \eta_{1}^{c}=\bar{M}_{1,3} \ddot{q}_{3}+\bar{C}_{13} \dot{q}_{3}+\eta_{1} \\
& \eta_{2}^{c}=\bar{M}_{2,3} \ddot{q}_{3}+\bar{M}_{2,4} \ddot{q}_{4}+\bar{M}_{2,5} \ddot{q}_{5}+\bar{C}_{23} \dot{q}_{3}+ \\
& +\bar{C}_{24} \dot{q}_{4}+\bar{C}_{25} \dot{q}_{5}+\eta_{2}
\end{aligned}
$$
(composed by the first elements of $C(q, \dot{q}))$ is such that $N_{1}=\dot{M}_{1}-2 C_{1}$ is a skew symmetric matrix (traducing the passivity property of the subsystem).

- $J_{1}^{T}=J^{T}\left(x_{11}\right) \in R^{(6 \times 12)}$ is the Jacobian matrix reduced to the system $\Sigma_{1}$ and $F_{1}$ the corresponding forces vector.

- $\nu_{1}$ represents the coupling terms du to dynamics of the other subsystems. These coupling terms affect a passive (sub) system. This is the key property of the proposed parametrization and the system decomposition in simpler subsystems. It highlights the interest of coupling terms coming from passive subsystems.

By using $x_{11}=\left(q_{1}, q_{2}\right)$ and $x_{12}=\left(\dot{q_{1}}, \dot{q_{2}}\right)$, an equivalent state space representation can be written:

$$
\begin{aligned}
& \dot{x}_{11}=x_{12} \\
& \dot{x}_{12}=M_{1}^{-1}\left(J_{1}^{T} F_{1}-C_{1} x_{12}-V_{12}\left(x_{11}, x_{12}\right)-\nu_{1}\right) \\
& y_{1}=h\left(x_{11}, x_{12}\right)
\end{aligned}
$$


2) Suspensions Dynamics $\Sigma_{2}$ : From the global equations we take the third one (equ 11).

$$
\begin{aligned}
F_{S} & =\bar{M}_{33} \ddot{q}_{3}+\bar{C}_{33} \dot{q}_{3}+V_{3}(q, \dot{q})+\eta_{c}^{3} \\
\eta_{c}^{3} & =\bar{M}_{31} \ddot{q}_{1}+\bar{M}_{32} \ddot{q}_{2}+\bar{C}_{32} \dot{q}_{2}+\eta_{3}
\end{aligned}
$$

Let $x_{2}=\left(x_{21}, x_{22}\right)=\left(q_{3}, \dot{q}_{3}\right)$, the state space representation of $\left(\Sigma_{2}\right)$ is then:

$$
\begin{aligned}
& \dot{x}_{21}=x_{22} \\
& \dot{x}_{22}=\bar{M}_{3,3}^{-1}\left(J_{2}^{T} F_{2}-\bar{C}_{33} x_{22}-V_{3}\left(x_{21}, x_{22}\right)-\nu_{2}\right) \\
& y_{2}=h\left(x_{2}\right)
\end{aligned}
$$

with $\left.\nu_{2}=\left[\eta_{c}^{3}-J_{2}^{T} F_{2}\right)\right]$ and $F_{S}=J_{2}^{T} F_{2}$

This subsystem can also be shown to be passive $\left(N_{2}=\right.$ $\dot{M}_{2}-2 C_{2}$ is a skew symmetric matrix.

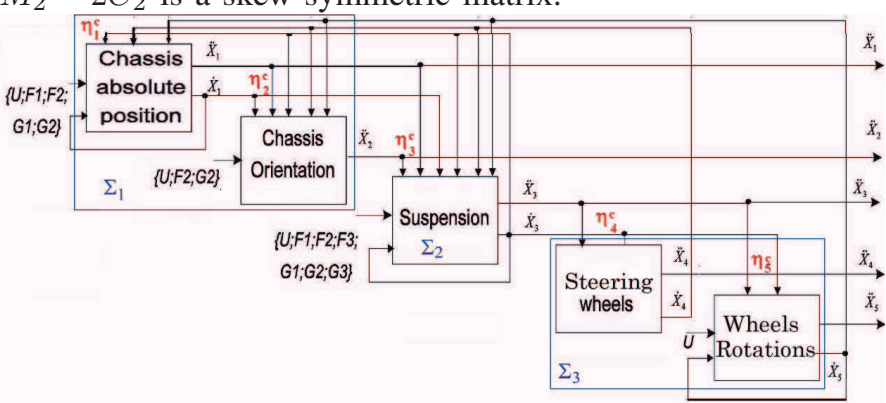

Figure 2. Five Passive sub Models for Robust Sliding Mode Observers

3) Wheels dynamics $\Sigma_{3}$ : The fastest dynamic in the vehicle model is the one of equation (7) is for wheels steering and rotations. The fourth equation of the model (1) stands for the steering front wheels with as inputs $U_{4}$. The motor / brake torque $U_{5}$ is applied to the two rear wheels.

$$
\begin{aligned}
& {\left[\begin{array}{l}
U_{4} \\
U_{5}
\end{array}\right]=} {\left[\begin{array}{ll}
\bar{M}_{4,4} & 0 \\
0 & \bar{M}_{5,5}
\end{array}\right]\left[\begin{array}{l}
\ddot{q}_{4} \\
\ddot{q}_{5}
\end{array}\right]+\left[\begin{array}{ll}
0 & \bar{C}_{4,5} \\
\bar{C}_{5,4} & 0
\end{array}\right]\left[\begin{array}{l}
\dot{q}_{4} \\
\dot{q}_{5}
\end{array}\right] } \\
&+ {\left[\begin{array}{l}
V_{4}\left(\dot{q}_{4}, \ddot{q}_{4}\right) \\
V_{5}\left(\dot{q}_{5}, \ddot{q}_{5}\right)
\end{array}\right]+\left[\begin{array}{l}
\eta_{c}^{4} \\
\eta_{c}^{5}
\end{array}\right] } \\
& q_{4}^{T}=\left[\delta_{3}, \delta_{4}\right] ; \text { and } \quad q_{5}^{T}=\left[\varphi_{1}, \varphi_{2}, \varphi_{3}, \varphi_{4}\right] \\
& \eta_{c}^{4}=\bar{M}_{42} \ddot{q}_{2}+\bar{C}_{42} \dot{q}_{2}+\eta_{4} \\
& \eta_{c}^{5}=\bar{M}_{52} \ddot{q}_{2}+\bar{C}_{52} \dot{q}_{2}+\eta_{5}
\end{aligned}
$$

By choosing $x_{31}=\left(q_{4}^{T}, q_{5}^{T}\right)^{T}$ and $x_{32}=x_{31}$, the equivalent state space representation can be written:

$$
\begin{aligned}
& \dot{x}_{31}=x_{32} \\
& \dot{x}_{32}=\bar{M}_{3}^{-1}\left(U_{45}-C_{3} x_{32}-V_{45}\left(x_{31}, x_{32}\right)-\nu_{3}\right) \\
& y_{3}=h\left(x_{31}, x_{32}\right)
\end{aligned}
$$

with $\nu_{3}=\left[\begin{array}{l}\eta_{c}^{4} \\ \eta_{c}^{5}\end{array}\right]$ and $U_{45}=\left[\begin{array}{c}U_{4} \\ U_{5}\end{array}\right]$

Then in conclusion, the vehicle can be presented as subsystems $\Sigma_{1}, \Sigma_{2}$ and $\Sigma_{3}$ corresponding respectively to chassis translations and rotations, Suspensions elongations, wheel steering and rotations (see figure 2). It is worthwhile to note that until now there are no approximations when considering the 5 equations. All the subsystems can be shown to fulfill the previously presented Passive Feedback Equivalent Scheme (PEFS). Approximations will be made when neglecting the coupling terms $\eta_{c}^{i}$. In the previous expressions, we remark that splitting the model is helpful, when using reduced models, to identify what is neglected regard to our proposed nominal model with 16 DoF. This is emphasized by the PEFS which show us that the main system properties are preserved and what is neglected do not change stability properties. $\eta_{c}^{i}$ are coupling terms du to connections with the other PEFS sub systems. We can verify that these terms are bounded such and as $\left|\eta_{c}^{i}\right|<k_{i} \forall t$.

4) Evaluation of the coupling terms in simulation: To evaluate the level of the coupling terms some simulation have been realized. We use in simulations, sinusoidal steering (with a period near to $5 \mathrm{~s}$ ) and a velocity near to $5 \mathrm{~m} / \mathrm{s}$ during 6 seconds and then decreasing (see figure 3 ).

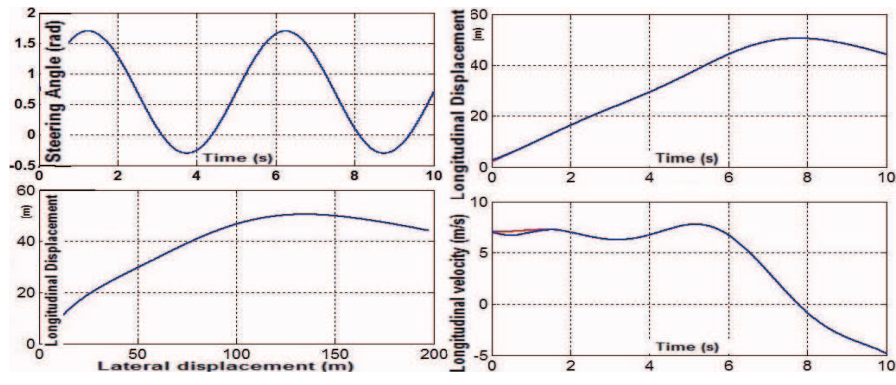

Figure 3. Vehicle behavior for a sinusoidal steering

Figure (4) show the 3 components of the coupling terms $\eta_{c}^{1}$ left column and $\eta_{c}^{2}$ on the right column. We can remark that they are very small and negligible compared to the inputs $F_{t}$ and $F_{r}$, drawn bellow in figure (5).
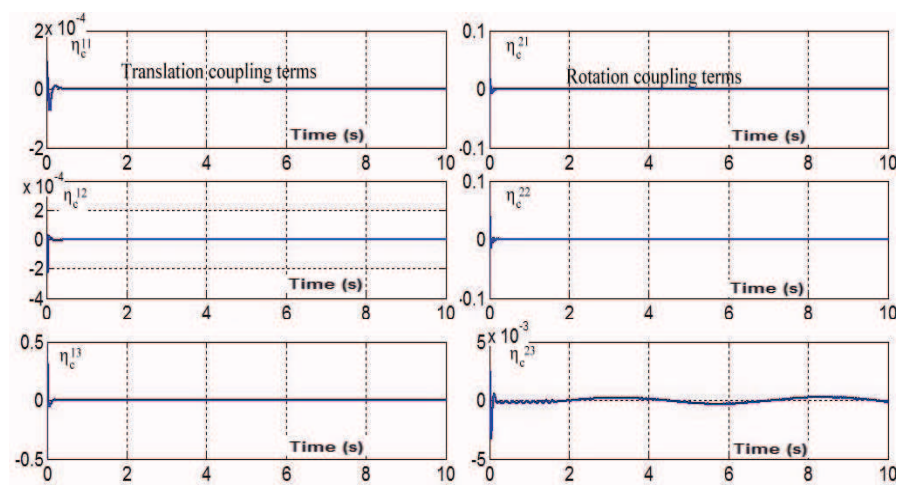

Figure 4. The components of the coupling terms $\eta_{c}^{1}$ left and $\eta_{c}^{2}$ right

The inputs $F_{t}$ and $F_{r}$ (figure 5) are located at the same place as the coupling terms, which are then matched perturbations. Figures (6) show the 4 components of the coupling terms $\eta_{c}^{3}$

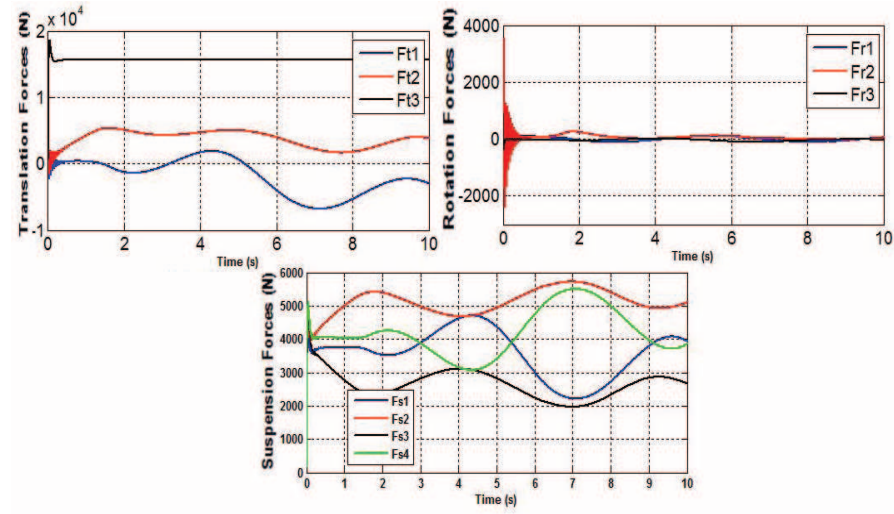

Figure 5. The inputs $F_{t}$ and $F_{r}$ of the 2 first blocks for sinusoidal steering 
appearing in the suspension block as perturbations. We remark also that they are very small and negligible compared to the inputs $F_{s_{1.4}}=J_{2}^{T} F_{2}$, drawn in the bottom of figure (5).
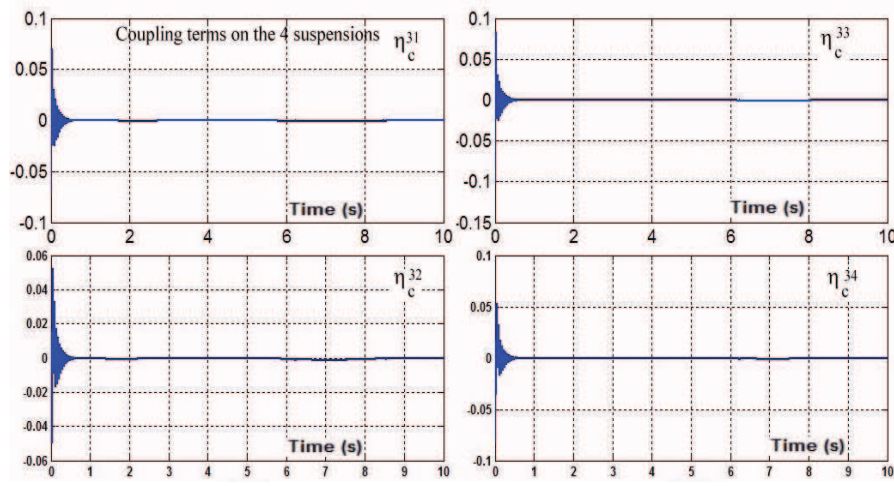

Figure 6. The VSAS suspension Coupling terms for sinusoidal steering

\section{OBSERVERS DESIGN}

The Sliding Mode Observers (SMO) technique is an attractive approach for its robustness and second for its finite time convergence feature. The latter is interesting to avoid use of costy sensors in complex systems. As we show in this work, to be able to estimate the unknown input forces and then adherence and road characteristics, we can use several steps of observations and estimations. Using partial state observers to get good and robust estimation of the vehicle state, using the previously presented blocks and splitting, we can consider estimation of the remaining variables in the process dynamics. This is done by filtering and estimating what have been considered as perturbations in the previous steps. This allowed us to avoid observability problems by using robust and cheap SMOs instead of sensors in a procedural estimation approach. In what follows, for the proposed observers, we consider the input forces unknown but slowly time varying. The assumption $\dot{F} \approx 0$ means that the changes in the forces are small in the mean as for example only one change with significant amplitude but during an intervall which is not too short. Adding a linearized model arround some operating point can enhance the estimations quality.

\section{A. First Order Sliding Mode Observers}

1) Observer for the chassis Dynamics $\Sigma_{1}:$ Estimations of the nominal functions $\hat{M}(q), \hat{C}(q, \dot{q}), \hat{V}(q, \dot{q}), \hat{G}(q)$ are assumed known. If not, some intermediate values can be considered to develop the following proposed observers. Let us note the states estimation errors $\tilde{x}_{i j}=\hat{x}_{i j}-x_{i j}$

The proposed observer for the chassis dynamics is:

$\hat{x}_{11}=\hat{x}_{12}-\Lambda_{11} \operatorname{sign}\left(\tilde{x}_{11}\right)$

$\dot{\hat{x}}_{12}=\hat{M}_{1}^{-1}\left(J_{1}^{T} \hat{F}_{1}-\hat{C}_{1}\left(\hat{x}_{11}, \hat{x}_{12}\right) \hat{x}_{12}-\hat{V}_{12}\right)-\Lambda_{12} \operatorname{sign}\left(\tilde{x}_{11}\right)$

$\dot{\hat{F}}_{1}=-P \Lambda_{13} \operatorname{sign}\left(\tilde{x}_{11}\right)$
The observation error dynamics is (see eq10 and 18):

$$
\begin{aligned}
\dot{\tilde{x}}_{11} & =\tilde{x}_{12}-\Lambda_{11} \operatorname{sign}\left(\tilde{x}_{11}\right) \\
\dot{\tilde{x}}_{12} & =\zeta_{1}-M_{1}\left(x_{11}\right)^{-1} J_{1}^{T}\left(x_{11}\right) \tilde{F}_{1}-\Lambda_{12} \operatorname{sign}\left(\tilde{x}_{11}\right) \\
\dot{\tilde{F}}_{1} & =-P \Lambda_{13} \operatorname{sign}\left(\tilde{x}_{11}\right) \\
\tilde{M}_{1}^{-1} & =\hat{M}_{1}^{-1}-M_{1}^{-1} ; \tilde{C}_{1}=\hat{C}_{1}-C_{1} ; \tilde{V}_{12}=\hat{V}_{12}-V_{12}
\end{aligned}
$$

with $\zeta_{1}$ as matched perturbation $\zeta_{1}=$ $M_{1}\left(x_{11}\right)^{-1}\left(C_{1}\left(x_{1}, x_{2}\right) x_{2}-\hat{C}_{1}\left(\hat{x}_{1}, \hat{x}_{2}\right) \hat{x}_{2}+\tilde{V}_{12}\left(\hat{x}_{1}, \hat{x}_{2}\right)+\right.$ $\left.\nu_{1}\right)+\tilde{M}_{11}^{-1}\left(x_{11}\right)\left(\hat{C}_{1}\left(\hat{x}_{1}, \hat{x}_{2}\right) \hat{x}_{2}+\hat{V}_{12}\left(\hat{x}_{1}, \hat{x}_{2}\right)-J_{1}^{T}\left(x_{11}\right) \hat{F}_{1}\right)$;

Estimation errors on forces are: $\tilde{F}_{1}=\hat{F}_{1}-F_{1}$ with $\zeta_{1}$ all neglected terms and remaining modeling and coupling errors. These can be assumed bounded owing to fact that all involved terms are either estimates or come from a passive mechanical part of the system and $\left|\nu_{1}\right|<\kappa_{0} \forall t \in \mathbb{R}_{+}$.

The Lyapunov function $V_{1}=\frac{1}{2} \tilde{x}_{11}^{T} \tilde{x}_{11}$, help to show that the sliding surface $\tilde{x}_{11}=0$ is attractive if we choose $\lambda_{12}^{i}$ such as $\left|\tilde{x}_{12}^{i}\right|<\lambda_{12}^{i}$ for $i=1, . ., 3$.

After a finite time $t_{01}$, we will get in average $\tilde{x}_{11}=0$ and $\dot{\tilde{x}}_{11}=0$. We obtain a reduced dynamic for the estimation error:

$$
\begin{aligned}
\dot{\tilde{x}}_{12} & =\zeta_{1}-M_{1}\left(x_{11}\right)^{-1} J_{1}^{T}\left(x_{11}\right) \tilde{F}_{1}-\Lambda_{12} \Lambda_{11}^{-1} \tilde{x}_{12} \\
\dot{\tilde{F}} & =-P \Lambda_{13} \Lambda_{11}^{-1} \tilde{x}_{12}
\end{aligned}
$$

For the second step of the convergence proof, consider $V_{2}\left(\tilde{x}_{12}, \tilde{F}_{1}\right)=\frac{1}{2} \tilde{x}_{12}^{T} \tilde{x}_{12}+\frac{1}{2} \tilde{F}_{1}^{T} P^{-1} \tilde{F}_{1}$ then $\dot{V}_{2}\left(\tilde{x}_{12}, \tilde{F}_{1}\right)$ becomes if we let $\Lambda_{13}=\left(M_{1}\left(x_{11}\right)^{-1} J_{1}^{T}\left(x_{11}\right)\right) \Lambda_{11}$

$$
\dot{V}_{2}=-\tilde{x}_{12}^{T} \Lambda_{12} \Lambda_{11}^{-1} \tilde{x}_{12}-\tilde{x}_{12}^{T} \zeta_{1}
$$

Now as previously choose $\lambda_{11}^{i}$ and $\lambda_{12}^{i}$ (the diagonal elements of the gain matrices $\Lambda_{11}, \Lambda_{12}$ ) large enough and $\Lambda_{13}=$ $\left(M_{1}\left(x_{11}\right) J_{1}^{T}\left(x_{11}\right)\right) \Lambda_{11}$. Then convergence of $\left(\hat{x}_{11}^{i}, \hat{x}_{21}^{i}\right)$ toward $\left(x_{11}^{i}, x_{21}^{i}\right)$ is obtained and estimation errors on forces are bounded.

2) Observer for Suspensions dynamics $\Sigma_{2}:$ We assume that the wheels are always in contact with the ground $(\xi=1)$ and note $\tilde{x}_{21}^{i}=\hat{x}_{21}^{i}-x_{21}^{i}$ and $\tilde{x}_{22}^{i}=\hat{x}_{22}^{i}-x_{22}^{i}$ the estimation errors and $\tilde{F}_{i 2}=\hat{F}_{i 2}-F_{i 2}$ force estimation error. The proposed observer, for each wheel suspension, is:

$$
\begin{aligned}
& \dot{\hat{x}}_{21}=\hat{x}_{22}-\lambda_{21} \operatorname{sign}\left(\tilde{x}_{21}\right) \\
& \dot{\hat{x}}_{22}=\hat{M}_{33}^{-1}\left(J_{2}^{T} \hat{F}_{2}-\hat{C}_{33}\left(\hat{x}_{21}, \hat{x}_{22}\right) \hat{x}_{22}-\hat{V}_{3}\left(\hat{x}_{21}, \hat{x}_{22}\right)\right)-\lambda_{22}^{i} \operatorname{sign}(\tilde{x}
\end{aligned}
$$

$\hat{F}_{i 2}=-P \lambda_{23}^{i} \operatorname{sign}\left(\tilde{x}_{21}\right)$

The observation error dynamics is (25-13):

$$
\begin{aligned}
& \dot{\tilde{x}}_{21}=\tilde{x}_{22}-\lambda_{21} \operatorname{sign}\left(\tilde{x}_{21}\right) \\
& \dot{\tilde{x}}_{22}=-\zeta_{2}+M_{33}^{-1}\left(J_{2}^{T}\left(x_{21}\right) \tilde{F}_{2}-K_{v} \tilde{x}_{22}\right)-\lambda_{22} \operatorname{sign} \tilde{x}_{21}
\end{aligned}
$$

$$
\tilde{F}_{i 2}=-P \lambda_{23} \operatorname{sign}\left(\tilde{x}_{21}\right)
$$


with $\zeta_{2}=M_{33}^{-1}\left(C_{33}\left(x_{21}, x_{22}\right) x_{2}-C_{33}\left(\hat{x}_{21}, \hat{x}_{22}\right) \hat{x}_{2}+\tilde{G}+\right.$ $\left.\nu_{2}\right)+\tilde{M}_{33}^{-1}\left(\left(K_{p} \hat{x}_{21}^{i}+C_{33}\left(\hat{x}_{21}, \hat{x}_{22}\right) \hat{x}_{2}+\hat{G}\right)\right.$

Like the previous case we choose $V_{1}=\frac{1}{2}\left(\tilde{x}_{21}^{T} \tilde{x}_{21}\right)$ and show that $\hat{x}_{12}$ converges to $x_{12}$ in finite time $t_{02}$ if we ensure that $\forall t>0$ that $\left|\tilde{x}_{22}\right|<\lambda_{21}$,. Then we deduce the reduced average dynamics $\tilde{x}_{22}=\lambda_{21} \operatorname{sign}\left(\tilde{x}_{12}\right)$

$$
\begin{aligned}
& \dot{\tilde{x}}_{22}=-\zeta_{2}-\bar{M}_{33}^{-1}\left(K_{v}+\lambda_{22} \lambda_{21}^{-1}\right) \tilde{x}_{22}+M_{33}^{-1} J^{T}\left(x_{21}\right) \hat{F}_{2} \\
& \dot{\tilde{F}}_{2}=-P \lambda_{23} \lambda_{21}^{-1} \tilde{x}_{21}
\end{aligned}
$$

Let $V_{2}=\frac{1}{2} \tilde{x}_{22}^{T} \bar{M}_{33}\left(x_{21}\right) \tilde{x}_{22}+\frac{1}{2} \tilde{F}_{2}^{T} P^{-1} \tilde{F}_{2}$, its derivative $\dot{V}_{2}$ becomes, if we take $\lambda_{23}=J^{T}\left(x_{21}\right) \lambda_{12}$,

$$
\dot{V}_{2}=-\tilde{x}_{2}^{T}\left(K_{v}+\lambda_{23} \lambda_{21}^{-1}\right) \tilde{x}_{22}^{i}-\tilde{x}_{2}^{T} \bar{M}_{2,2} \zeta_{2}
$$

We can conclude as previously that if we choose $\lambda_{21}$ and $\lambda_{22}$ (the diagonal elements of the gain matrices $\Lambda_{21}$ et $\Lambda_{22}$ ) large enough and $\Lambda_{23}=J^{T} \Lambda_{21}$ then convergence of $\left(\hat{x}_{21}, \hat{x}_{22}\right)$ toward $\left(x_{21}, x_{22}\right)$ is obtained and estimation errors on forces $\tilde{F}_{2}$ remains only bounded.

3) Observer for Wheels Dynamics $\Sigma_{3}$ : For each wheel $i$, the proposed observer is as follows:

$$
\begin{aligned}
& \dot{\hat{x}}_{31}^{i}=\hat{x}_{32}^{i}-\lambda_{31}^{i} \operatorname{sign}\left(\hat{x}_{31}^{i}-x_{31}^{i}\right) \\
& \dot{\hat{x}}_{32}=J^{-1}\left(\tau-r \hat{F}_{3 i}\right)-\lambda_{32}^{i} \operatorname{sign}\left(\hat{x}_{31}^{i}-x_{31}^{i}\right) \\
& \hat{F}_{3 i}=-P \lambda_{33}^{i} \operatorname{sign}\left(\hat{x}_{31}^{i}-x_{31}^{i}\right)
\end{aligned}
$$

The torque $\tau$ is assumed known. This observer can be easily extended to estimate the torque by adding an equation defining the drive line producing the torque. Observation error dynamics is then:

$$
\begin{aligned}
& \dot{\tilde{x}}_{31}^{i}=\tilde{x}_{32}^{i}-\lambda_{31}^{i} \operatorname{sign}\left(\tilde{x}_{31}^{i}\right) \\
& \dot{\tilde{x}}_{32}=-r J^{-1} \tilde{F}_{3 i}-\lambda_{32}^{i} \operatorname{sign}\left(\tilde{x}_{31}^{i}\right)+\eta_{c}^{3 i} \\
& \dot{\tilde{F}_{3 i}}=-P \lambda_{33}^{i} \operatorname{sign}\left(\tilde{x}_{31}^{i}\right)
\end{aligned}
$$

with $\tilde{x}_{31}^{i}=\hat{x}_{31}^{i}-x_{31}^{i}, \tilde{x}_{32}^{i}=\hat{x}_{32}^{i}-x_{32}^{i}$ the errors on estimations of states $x_{3}^{i}$ and forces $\tilde{F}_{3 i}=\hat{F}_{3 i}-F_{3 i}$.

We can prove the convergence in finite time $\left(t_{03}\right)$ of states estimates $\hat{x}_{31}^{i}$ and bounded of forces estimation by using the Lyapunov functions $V_{1}=\frac{1}{2}\left(\tilde{x}_{11}^{i}\right)^{2}$, and $V_{2}=\frac{1}{2}\left(\tilde{x}_{21}^{i}\right)^{2}+$ $\frac{1}{P}\left(\tilde{F}_{i 1}\right)^{2}$.

Let us note $\xi^{T}=\left[\tilde{x}_{21}^{i}, v_{c}^{1 i}\right]$ then $\dot{V}_{2}$ become upper bounded

$$
\begin{aligned}
& \dot{V}_{2}=-\xi^{T} Q \xi+\left(v_{c}^{1 i}\right)^{2}=-\xi^{T}\left[\begin{array}{cc}
\frac{\lambda_{21}^{i}}{\lambda_{11}^{i}} & -\frac{1}{2} \\
-\frac{1}{2} & 1
\end{array}\right] \xi+\left(v_{c}^{1 i}\right)^{2} \\
& \dot{V}_{2} \leq-\lambda_{\inf }\{Q\}\|\xi\|^{2}+\left\|v_{c}^{1 i}\right\|^{2} \\
& \dot{V}_{2} \leq-\lambda_{\inf }\{Q\}\left\|\tilde{x}_{21}^{i}\right\|^{2}+\left(1-\lambda_{\inf }\{Q\}\right)\left\|v_{c}^{1 i}\right\|^{2}
\end{aligned}
$$

then $\tilde{x}_{21}^{i}$ converges to 0 if $\lambda_{21}^{i}$ is such as $\lambda_{\inf }\{Q\}$ is large enough. $\lambda_{11}^{i}$ and $\lambda_{21}^{i}$ large enough give convergence of $\left(\hat{x}_{11}^{i}, \hat{x}_{21}^{i}\right)$ toward $\left(x_{11}^{i}, x_{21}^{i}\right)$ and errors $\tilde{F}_{i 1}$ remain bounded.

\section{B. High Order Sliding Mode Observers}

In this part we will use a High Order Sliding Mode observers (HOSM See [14]) to deduce our estimations. The HOSM dynamics converge in finite time.

1) Observer for the chassis Dynamics $\Sigma_{1}$ : The proposed observer is the following (with $\tilde{x}_{11}=\hat{x}_{11}-x_{11}$ ):

$$
\begin{gathered}
\dot{\hat{x}}_{11}=v_{11}=\hat{x}_{12}-\lambda_{11}\left|\hat{x}_{11}-x_{11}\right|^{\frac{2}{3}} \operatorname{sign}\left(\hat{x}_{11}-x_{11}\right) \\
\dot{\hat{x}}_{12}=-\zeta_{12}-\lambda_{12}\left|\hat{x}_{12}-v_{11}\right|^{\frac{1}{2}} \operatorname{sign}\left(\hat{x}_{12}-v_{11}\right) \\
\text { with } \zeta_{12}=\hat{M}_{11}^{-1}\left(\hat{C}_{1}\left(\hat{x}_{1}, \hat{x}_{2}\right) \hat{x}_{2}+\hat{G}-J_{1}^{T} \hat{F}\right)
\end{gathered}
$$

\section{Simulation Results}

The simulator previously developed by our staff (SimK106N, available on demand to first author) is used in order to test and validate the proposed observers and our approach of model splitting and developing partial state estimators. The used parameters and environment characteristics have been validated in a previous work in collaboration with the LCPC [2][7][5].

The simulation results presented are obtained for a driving with sinusoidal steering command of $20 \mathrm{deg}$ amplitude. The results are good for the First Order Sliding Mode Observers (see figure 7) and also for HOSM based observers (see figure 8 ); The two kind of observers may give very good results by adjusting the gain parameters.

\section{CONCLUSION}

In this paper, we have proposed efficient and robust observers allowing to estimate states and unknown inputs (torques or forces). The model formulation has been done such that the passivity property is preserved. This copes well for SMO approach and avoid chattering problems.

These observers obey to the first kind assuming that input forces and torques are constant or slowly time varying ( $\dot{F} \simeq 0$ ). The robustness of the sliding mode observer versus uncertainties on model parameters is an important feature. First and Second Order Sliding Mode Observers have been developed and their performance evaluated. These observer are illustrated by simulation results to show effectiveness of their performance. These results validate the proposed observers and our approach of model splitting and developing partial state estimators. .

\section{ACKNOWLEDGMENTS}

This work has been done in the context of a the GTAA (Groupe Thématique Automatique et Automobile). Acknowledgments are also addressed to Y. Delanne and L. Fridman for the useful discussions.

\section{REFERENCES}

[1] R. A. Ramirez Mendoza. Sur la modélisation et la commande des véhicules automobiles. doctorat de l'institut national polytechnique de grenoble. 1997.

[2] N. K. M'Sirdi J.C. Cadiou et Y. Delanne A. El Hadri, G. Beurier. Simulation et observateurs pour l'estimation des performances dynamiques d'un vhicule. CIFA, 2000.

[3] G. Venture. Identification des paramètres dynamiques d'une voiture. Thèse de Doctorat de l'Ecole Polytechnique de l'Université de Nantes, IRCCyN. Nantes, novembre, 2003. 

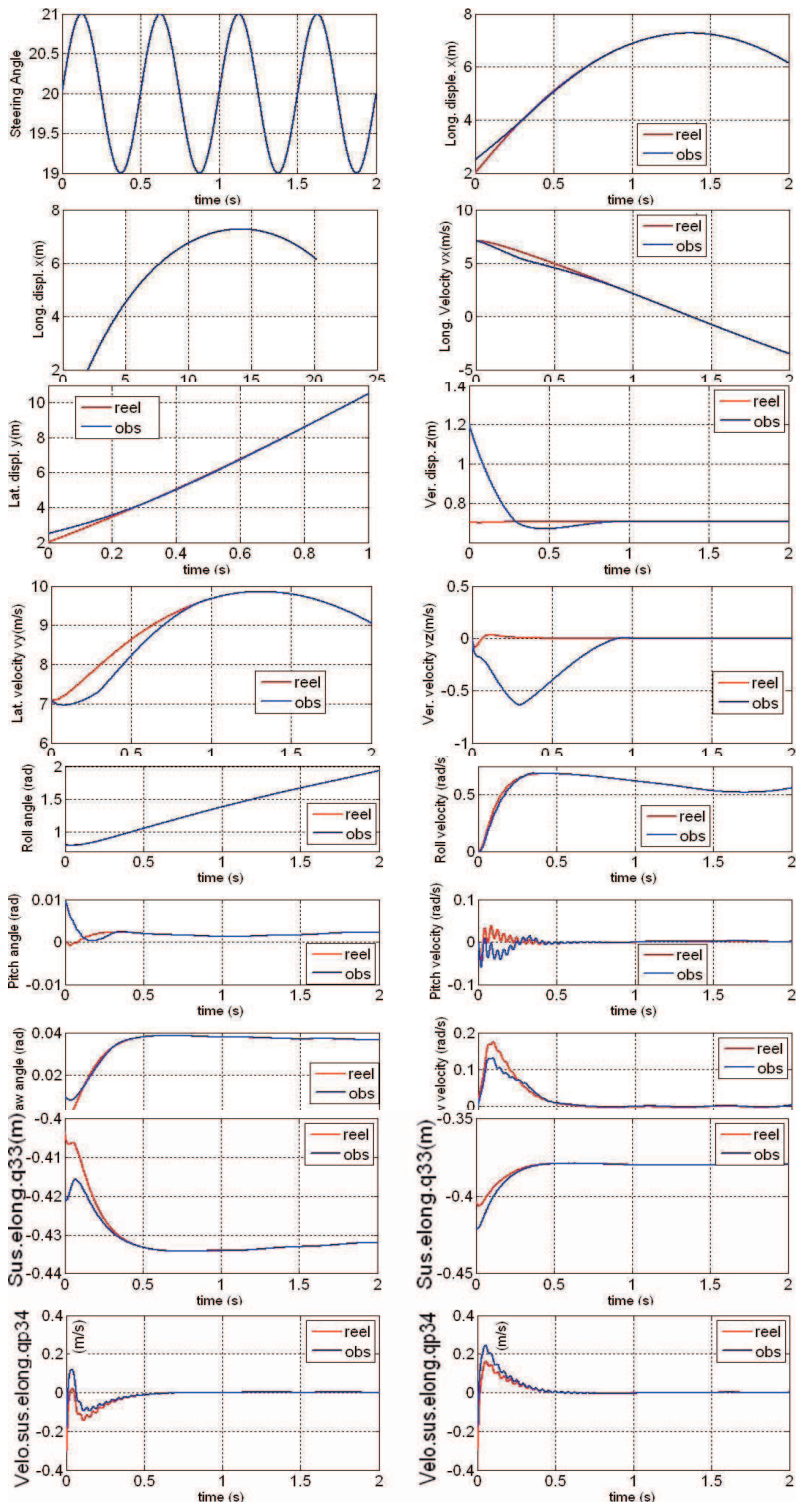

Figure 7. Results of the Partial state Observer FOSM

[4] K. N. M'sirdi, L. H. Rajaoarisoa, J.-F. Balmat, and J. Duplaix. Modelling for control and diagnosis for a class of non linear complex switched systems. Advances in Vehicle Control and Safety AVCS 07, Buenos Aires, Argentine, February 8-10, 2007.

[5] A. Rabhi, H. Imine, N. M'Sirdi, and Y. Delanne. Observers with unknown inputs to estimate contact forces and road profile. AVCS'04, International Conference on Advances in Vehicle Control and Safety Genova -Italy, October 28-31, 2004.

[6] N.K. M'sirdi, A. Rabhi, L. Fridman, J. Davila, and Y. Delanne. Second order sliding mode observer for estimation of velocities, wheel sleep, radius and stiffness. ACC, Proceedings of the 2006 American Control Conference Minneapolis, Minnesota, USA, June 14-16,:3316 -3321, 2006.

[7] N.K.M'sirdi, A.Rabhi, N.Zbiri, and Y. Delanne. Vrim: Vehicle road interaction modelling for estimation of contact forces. Tyre Colloquium Tyre Models For Vehicle Dynamics Analysis, 2004.

[8] A. Rabhi L. Fridman N. K. M"Sirdi, A. Boubezoul. Estimation of performance of heavy vehicles by sliding modes observers. ICINCO:pp360$365,2006$.

[9] N.K. M'Sirdi, A. Rabhi, and Aziz Naamane. Vehicle models and estimation of contact forces and tire road friction. ICINCO, Invited paper ICINCO:351-358, 2007.

[10] K. N. M'Sirdi, A. Rabhi, and A. Naamane. A nominal model for vehicle dynamics and etimation of input forces and tire friction. Conference on control systems, Proceedings of the CSC 07, Marrakech, Maroc, 16-18 mai 2007., 2007.
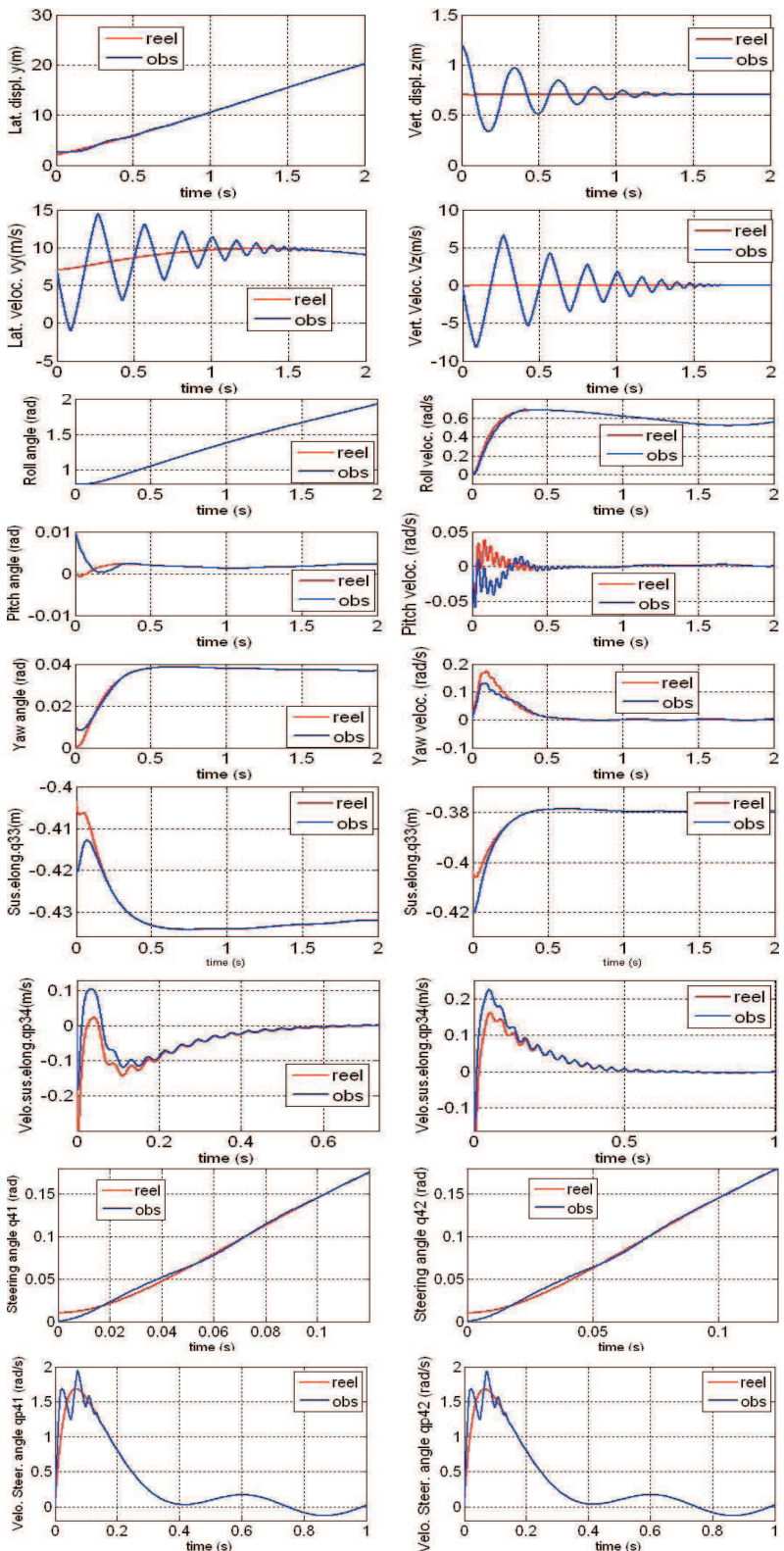

Figure 8. Results of the HOSM Observers

[11] S. Arimoto and F. Miyazaki. Satability and robustness of PID feedback control for robot of sensory capability. In 3rd Int. Symp. Of Robotics Resarch, Gouvieux, France, 1985.

[12] E.Velenis M.Basset G.Gissinger C.Canudas de Wit, P.Tsiotras. Dynamic friction models for road/tire longitudinal interaction. Vehicle Syst. Dynamics, 39(3):189-226, 2003.

[13] N. K. MSirdi, B. Jaballah, A. Naamane, and H. Messaoud. Robust observers and unknown input observers for estimation, diagnosis and control of vehicle dynamics. IEEE/RSJ International Conference on Intelligent RObots and Systems, Invited paper in the Workshop on Modeling, Estimation, Path Planning and Control of All Terrain Mobile Robots, IROS 08:September 22th 2008, Nice, France. http://wwwlasmea.univbpclermont.fr/MEPPC08/, 2008.

[14] A. Levant. Hight-order sliding modes, differentiation and outputfeedback control. International Journal of Control, 76:924-941, 2003. 